\title{
A Low-Complexity ECG Feature Extraction Algorithm for Mobile Healthcare Applications
}

\author{
Evangelos B. Mazomenos*, Dwaipayan Biswas, Amit Acharyya, Taihai Chen, \\ Koushik Maharatna, Member, IEEE, James Rosengarten, John Morgan and Nick Curzen
}

\begin{abstract}
This paper introduces a low-complexity algorithm for the extraction of the fiducial points from the Electrocardiogram (ECG). The application area we consider is that of remote cardiovascular monitoring, where continuous sensing and processing takes place in low-power, computationally constrained devices, thus the power consumption and complexity of the processing algorithms should remain at a minimum level. Under this context, we choose to employ the Discrete Wavelet Transform (DWT) with the Haar function being the mother wavelet, as our principal analysis method. From the modulus-maxima analysis on the DWT coefficients, an approximation of the ECG fiducial points is extracted. These initial findings are complimented with a refinement stage, based on the time-domain morphological properties of the ECG, which alleviates the decreased temporal resolution of the DWT. The resulting algorithm is a hybrid scheme of time and frequency domain signal processing. Feature extraction results from 27 ECG signals from QTDB, were tested against manual annotations and used to compare our approach against the state-of-the art ECG delineators. In addition, 450 signals from the 15-lead PTBDB are used to evaluate the obtained performance against the CSE tolerance limits. Our findings indicate that all but one CSE limits are satisfied. This level of performance combined with a complexity analysis, where the upper bound of the proposed algorithm, in terms of arithmetic operations, is calculated as $2.423 N+214$ additions and $1.093 N+12$ multiplications for $N \leq 861$ or $2.553 N+102$ additions and $1.093 N+10$ multiplications for $N>861$ ( $N$ being the number of input samples), reveals that the proposed method achieves an ideal trade-off between computational complexity and performance, a key requirement in remote CVD monitoring systems.
\end{abstract}

Index Terms-Mobile Healthcare, ECG Feature Extraction, Discrete Wavelet Transform, Low Complexity Algorithm

\section{INTRODUCTION}

Manuscript received May 25 2012; revised Novemeber 10 2012; accepted November 21 2012; Date of publication date of current version. This work was supported by E.U. ARTEMIS Joint Undertaking under the Cyclic and person-centric Health management: Integrated appRoach for hOme, mobile and clinical eNvironments - (CHIRON) Project under Grant Agreement \# 2009-1-100228.

E.B. Mazomenos, D. Biswas, T. Chen and K. Maharatna are with the ECS School, University of Southampton, SO17 1BJ, U.K. (e-mail: \{ebm,db9g10,tc10g09,km3\}@ecs.soton.ac.uk)

A. Acharyya is with EE Dept., IIT Hyderabad, India. (email: amit_acharyya@iith.ac.in)

J. Rosengarten, J. Morgan and N. Curzen are with the Southampton University Hospitals NHS Trust, Southampton, Hampshire, SO16 6YD, UK. and also with the University of Southampton, Southampton, SO17 1BJ, U.K. (email: james@ rosengarten.co.uk, jmm@ @rclinic.org, nick.curzen@suht.swest.nhs.uk)

Color versions of one or more of the figures in this paper are available online at http://ieeexplore.ieee.org.

Digital Object Identifier 10.1109/TITB.2012.2231312
GING population and continuous prevalence of Cardio$A$ vascular Diseases (CVD) - the number one cause of death (30\% of the global total of all deaths) according to the World Health Organization (WHO) - leading to long-term conditions, have put current healthcare systems worldwide under serious strain in terms of the quality of care delivery and its associated cost [1]. Coupled with the ensuing productivity loss, this leads to a formidable socio-economic challenge. Effective disease management through continuous monitoring and information fusion of vital physiological signals in chronic CVD patients is viewed as the key mechanism for the drastic reduction of the cost of CVD care delivery, the enhancement of the quality of care/life and the significant decrease in deaths and hospitalizations. Recently, advances in Wireless Sensor Network (WSN) technology enabled the development of the next-generation remote CVD monitoring and management systems, able to monitor the patients' vital sign data continuously in nomadic environment. The main approach is to use a number of battery powered wireless sensors to capture the vital signs and transmit all data to a centralized service for further analysis and disease prognosis. Since the traditional clinical feature extraction algorithms and information fusion techniques are, from a computational perspective, very intensive tasks, these parts are typically executed in main-frame type computational facilities. However, a significant energy expenditure component in such systems, is the energy required by the radio front-end for supporting continuous data transmission, which may not allow a long-term sustainable operation. Taking as an example, the ECG signal - the fundamental component of a remote CVD monitoring system - captured at $1 \mathrm{KHz}$ sampling rate with 16-bit quantization. Considering a typical Bluetooth V2 transceiver with 40 - $55 \mathrm{~mA}$ current consumption in transmission mode and a battery capacity of $1200 \mathrm{mAh}$ (the typical batteries used for WSN applications) and following the analysis presented in [2], we conclude that continuous data transmission can be supported only for $24 \mathrm{hrs}$. In addition to this, A/D conversion, quantization and signal preprocessing steps are also carried out at the sensor node and including those factors, it can be argued that the operation of a continuous transmission-based system, may not be realistically sustainable for more than $8-12 \mathrm{hrs}$. This falls well below the actual clinical notion of continuous monitoring in the sense of clinical usefulness.

The alternative approach for alleviating this problem - the approach adopted in the ARTEMIS Joint Undertaking funded CHIRON project of which the current work is a part - is to carry out the feature extraction and information fusion 
operations, used to attain clinical diagnosis that are supposed to be carried out at the main frame, at the sensor node itself and transmit the clinically relevant decisions to the centralized facility only upon request [3]. In this approach, the clinical results are stored on the sensor node itself and transmission takes place in burst mode at pre-set intervals or on-demand, therefore eliminating the requirement for continuous transmission while maintaining the notion of continuous monitoring. However, it may be argued that since the signal processing tasks required for such an approach are computationally intensive, the energy requirements for processing could overwhelm the energy required for continuous transmission. A recent attempt to implement an automated ECG delineation algorithm in a WSN node [4] indicated that the energy required for the ECG delineation is still significantly lower compared to the continuous transmission scenario, although it is pointed out that the selection of an appropriate algorithm, in terms of computational complexity and the type of microcontroller on which these operations will be executed, is of paramount importance in such a scenario.

Inspired by this fact, in this paper we propose a novel automated ECG feature extraction algorithm that is specifically designed to be efficient from a computational perspective, thus ideal for ultra-low-energy implementation. It is our belief that the proposed solution is suitable for integration in the latest generation remote CVD monitoring systems. It is important to point out that from a clinical application perspective, the main purpose of the automated ECG analysis in such remote monitoring systems, is to produce an "alarm signal" if some abnormality is detected over a long period of time and by no means perform any detailed diagnosis of the patient's clinical condition, as this is eventually done through more elaborate diagnostic means (e.g. imaging techniques) in clinical settings. This fact prompts us to trade-off accuracy and energy consumption although we show that the proposed system satisfies the CSE Working Party recommended tolerance limits [5] or is very close to them, when tested on standard databases, available in Physionet [6]. In addition we have tested the proposed method on 12 conventional paper ECG signals supplied by the Southampton General Hospital Cardiology Department and the achieved accuracy in all cases is endorsed by expert cardiologists. The overall system consumes a mere $269 \mathrm{nW}$ when synthesized in ST130 nm technology with 1.08 $\mathrm{V}$ supply voltage which clearly demonstrates its potential for low-power implementation.

The rest of the paper is structured as follows: after reviewing existing works in Section II, we describe our algorithm in Section III and provide an analysis of its computational complexity in Section IV. Section V is devoted for the validation of the algorithm, whereas conclusions are drawn in Section VI.

\section{BACKGROUND AND MOTIVATION}

Traditionally, automated ECG analysis either takes place online on high-performance bedside devices which are bulky, or is done offline on ambulatory recordings provided by an ECG data logger, like the Holter device. The main task here is to automatically detect clinically important ECG fiducial points like the onset and offset of the QRS complex, $\mathrm{P}$ and $\mathrm{T}$ waves. These are then used to calculate various ECG parameters like the RR-interval, the QRS-length, the PR-interval and the elevation/depression of the ST-segment. A plethora of excellent algorithms have been developed for such purpose based on different signal processing approaches, like the time-domain morphology analysis augmented by different types of filtering [7]-[13], artificial neural networks [14], pattern matching [15], hidden Markov models [16], Independent Component Analysis (ICA) [17] and combinations of the above mentioned methods [18]-[23]. Another significant line of approach is based on the Wavelet Transform (WT) which represents a signal in time-scale domain. Since the ECG signal is characterized by a periodic occurrence of patterns with different frequency contents, WT is deemed to be an excellent tool for its analysis. Subsequently, WT has been used extensively in the past [24][32]. In majority, WT-based ECG analysis algorithms use the quadratic spline wavelet as the basis function and employ the modulus-maxima analysis (MMA) method for finding out the peaks of characteristic waves at different resolution scales [33].

All the above mentioned algorithms show excellent performance in identifying the ECG fiducial points. However, the major concern associated with these algorithms is that they are extremely computationally intensive. As long as the operation is performed online on bulky bed-side devices or offline on a PC, their effectiveness is undoubted. But owing to the resource constrained nature - in particular, their limited battery life - of wearable wireless platforms these approaches may not be applicable, as the energy consumption is highly dependent on the computational complexity of the algorithmic process. Recently the WT-based ECG delineation algorithm proposed in [34] has been implemented on a commercially available Shimmer embedded sensor node which consists of an MSP430 microcontroller [35], showing that increased CPU activity leads to shorter node life-time. That work concludes that a careful study is necessary when choosing or designing a microcontroller [4]. However, the fundamental aspect of high computational complexity of the employed algorithms and the very fact that any general-purpose processor consumes several orders of magnitude more energy than an equivalent ASIC, leads us to believe that the sensor node should be augmented with an ASIC, dedicated for the ECG analysis which needs to be designed following a holistic algorithmarchitecture optimization approach. Therefore, in the present work we concentrated on developing a low-complexity algorithm, ideal to be realized as a standalone ASIC, by exploiting the advantages of both WT and time-domain morphology analysis, which is described in the following sections.

\section{Algorithmic Formulation}

The main engineering requirement for our purpose is to develop a low-complexity algorithm for the automated ECG analysis leading to low-power consumption. On the other hand, the clinical requirement is that the algorithm needs to produce results of acceptable clinical quality. These two seemingly contradictory criteria put major constraints on the selection of the signal processing tools that can be employed, in the 
backdrop of their physical implementability for reducing the energy consumption. However, the very fact that the ECG in such systems is mainly intended for generating an alarm allows one to trade-off algorithmic accuracy, while still staying within the clinically acceptable levels, for energy consumption. In the following subsections we will present the step-by-step formulation of a novel algorithm satisfying this criterion.

\section{A. Choice of the Wavelet function}

Owing to the effectiveness of WT, we base our algorithm on this approach. The biggest advantage of WT is that because of its time-scale analysis nature, it is inherently able to separate noise and artifacts, like isoelectric line wandering, at its different resolution levels as already been shown in [34]. However, the mother wavelet used for this purpose is a computationally demanding quadratic-spline Wavelet and as mentioned previously, has an impact on the energy consumption.

To keep the computational complexity low, we have selected the Haar wavelet - the simplest wavelet function. Although this function has its own limitations, we hypothesized that it still may be sufficient for the present purpose. The Haar Wavelet function and its corresponding scaling function are depicted in Fig. 1.

To ascertain the effectiveness of the Haar DWT in dealing with noise and isoelectric line wandering, 450 ECG signals sampled at $1 \mathrm{KHz}$, were investigated from the PTB database (PTBDB) [6]. Representative examples of signals that demonstrate isoelectric line wandering and signals that contain a significant amount of noise are illustrated alongside the 5 decomposition scales of Haar DWT in Fig. 2. It is obvious that significant noise components exist in the first two resolution levels. Therefore, applying MMA on these scales could lead to less accurate results. From our observations we concluded that by employing the MMA on the $2^{3}$ scale detailed DWT coefficients (cD_13), an initial estimation of the QRS fiducial points, within the PQRST-complex, is possible. This is justified from Fig. 2 where in the $2^{3}$ scale, noise components are suppressed to such a degree allowing for a noise-free representation of the ECG signal from the $2^{3}$ scale onwards. Therefore, for identifying the ECG waves it is sufficient only to consider the $2^{3}$ (for QRS) and $2^{5}$ (for P/T waves) resolution scales. This implicitly means substantial reduction in the computational complexity. Nevertheless, operating exclusively on the $2^{3}$ scale introduces the disadvantage of diminished temporal resolution due to downsampling. This is expected to add inaccuracies in the estimation of the QRS parameters. In addition, depending on the noise power and the statistical properties of the ECG signal, valid ECG frequency components may lie on the other resolution scales as well. Since we opt to operate on a single resolution scale these components will be discarded introducing further errors. Against this, our strategy is to refine the findings of the $2^{3}$ scale MMA, by introducing a computationally efficient time-domain morphology based compensatory step. This approach that we follow is ultimately justified by the obtained performance results (see Section V).

In a similar fashion, for the extraction of the P,T-waves parameters, instead of looking in multiple resolution levels we focus entirely on the $2^{5}$ scale. The reason for choosing the $2^{5}$ scale is that the effect of the isoelectric line wandering in the $2^{5}$ scale is minimal, as Fig. 2(a) illustrates and the $\mathrm{P}, \mathrm{T}$ components are amplified in the $2^{5}$ scale, compared to the $2^{4}$ scale, enabling for a robust approximation of the P,T boundaries, considering that the QRS boundaries are already obtained. Fig. 2(c) depicts an extreme case where both noise and isoelectric line wandering are present in the ECG sample. Again, in the $2^{3}$ and $2^{5}$ scales of DWT decomposition, the prominent coefficients represent valid QRS-complexes and P,T waves which can be captured with the use of MMA. The reason for choosing a higher scale for the $\mathrm{P}, \mathrm{T}$ waves is due to the fact that these waves comprise of lower frequency components than the QRS complex. The frequency response of the Haar DWT in the $2^{3}$ and $2^{5}$ scale is depicted in Fig. 3.
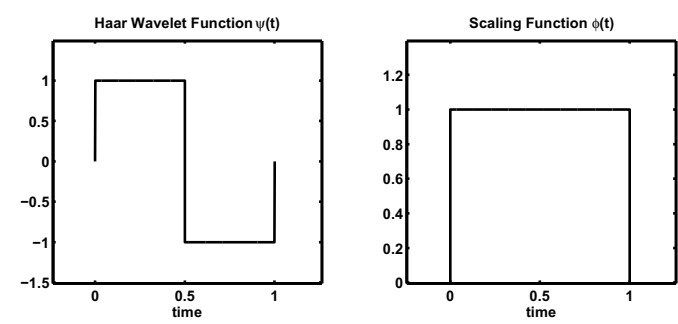

Fig. 1. Haar Wavelet and Scaling Functions

Contrary to our approach, other WT-based approaches conducted MMA on multiple-resolution levels. This multiple level MMA, coupled with the use of computationally intensive mother wavelets can yield very accurate results for ECG delineation [27], [34]. However we will demonstrate in our analysis (see Section IV) that the computational complexity, in terms of the arithmetic operations required, by the state of the art WT-delineator [34], which sets the basis of of DWTbased ECG feature extraction, far exceeds the computational complexity of the proposed algorithm, even after including the TDM step. This, in combination with the obtained performance (see Section V) in a diverse set of ECG signals prompt us to conclude that the selection of the Haar function as the mother wavelet and the choice of operating exclusively on one DWT resolution scale, augmented by the TDM refinement step, enable the proposed algorithm to achieve the desired trade-off of computational complexity and accuracy.

\section{B. DWT-based Initial Estimation}

The proposed feature extraction method operates on a single isolated heartbeat (PQRST-complex) and initially assumes the presence of all the constituent ECG waves (P,QRS,T). Our method is a combination of the MMA applied on the DWT decomposition levels and the time-domain morphological analysis of the ECG signal. Since we employ both frequency and time domain analysis we refer to the resulting algorithm as Hybrid Feature Extraction Algorithm (HFEA). To begin with, DWT decomposition takes place on the PQRST-complex. The analysis is performed at five dyadic space scales $\left(2^{1} \cdots 2^{5}\right)$ using the $2^{3}$ and $2^{5}$ scale for the extraction of QRS and 


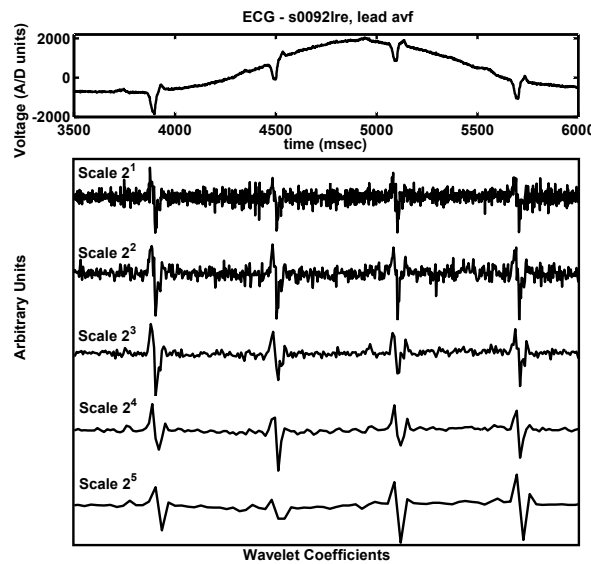

(a)

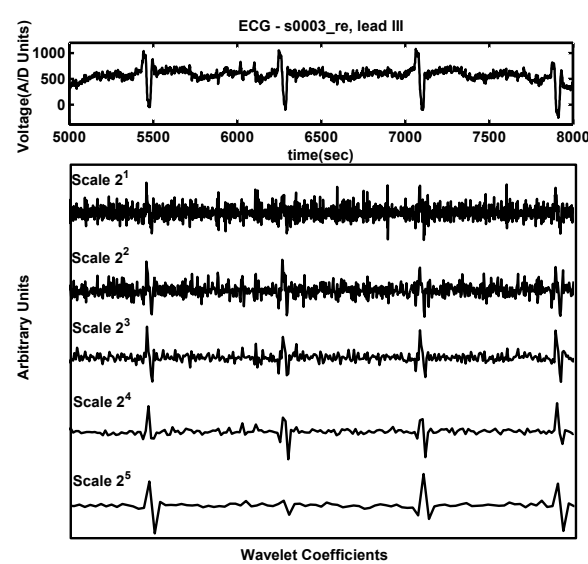

(b)

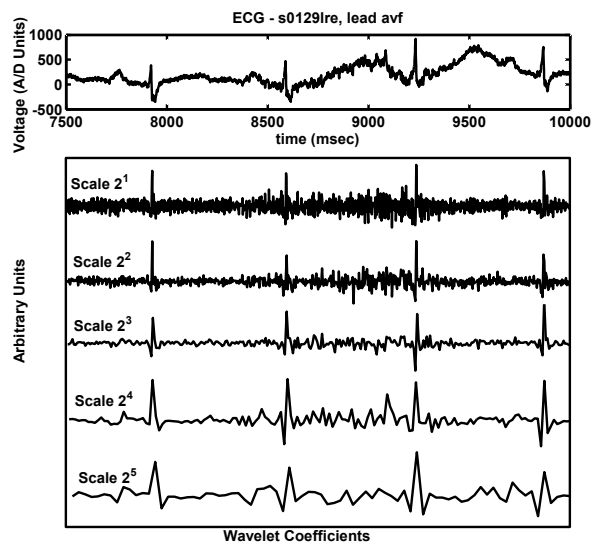

(c)

Fig. 2. ECG samples that (a) demonstrate isoelectric line wandering (b) are corrupted with noise (c) both, and their Haar DWT decomposition in 5 dyadic scales.

P/T waves parameters respectively. The multi-scale DWT decomposition is implemented as a cascade filter-bank structure (known as Mallat's Algorithm), illustrated in Fig. 4 featuring high- and low-pass filters. Downsampling is performed after filtering, to remove redundancy. The output of the high-pass filters $(\mathrm{H} 1(\mathrm{z}))$ provides the detailed WT coefficients $\left(\mathrm{cD} \_1 \mathrm{x}\right)$ at the $2^{\mathrm{x}}$ scale, while the approximate WT coefficients (cA_lx) are obtained from the output of the low-pass filters $(\mathrm{H} 0(\mathrm{z}))$. The frequency resolution increases in higher resolution scales, thus low frequency components are more easily detectable in high resolution scales $\left(2^{4}, 2^{5}\right)$. On the other hand, high frequency components are expected to be more distinguishable in the lower scales $\left(2^{1}, 2^{2}\right)$.

From the Haar transfer functions, it can be seen that the output of the high pass filter is proportional to the local averages of the derivative of the input, which in turn is a filtered version of the original signal. From this, it is established that potential extrema in the original signal $x[n]$ are represented as zero-crossing points in the $\mathrm{cD}_{-} \mathrm{lx}$ (where $\mathrm{x}$ is odd), while instances with maximum slope (deflection points) in the signal are transformed into extrema (minima or maxima) points on the cD_lx. The resolution scales $\left(2^{3}, 2^{5}\right)$ that we have chosen to operate on, satisfy the above rule.

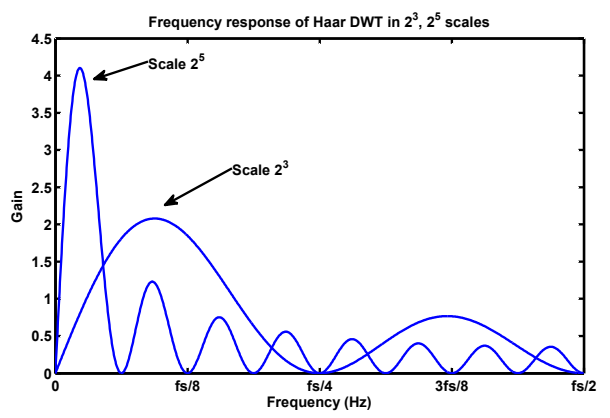

Fig. 3. Frequency response of the Haar DWT in scales $2^{3}$ and $2^{5}$, where $f_{s}$ is the input's sampling frequency

Once wavelet coefficients are computed, the focus turns on the $2^{3}$ scale detailed coefficients, where MMA is employed for the approximation of the zero-crossing points. Based on the

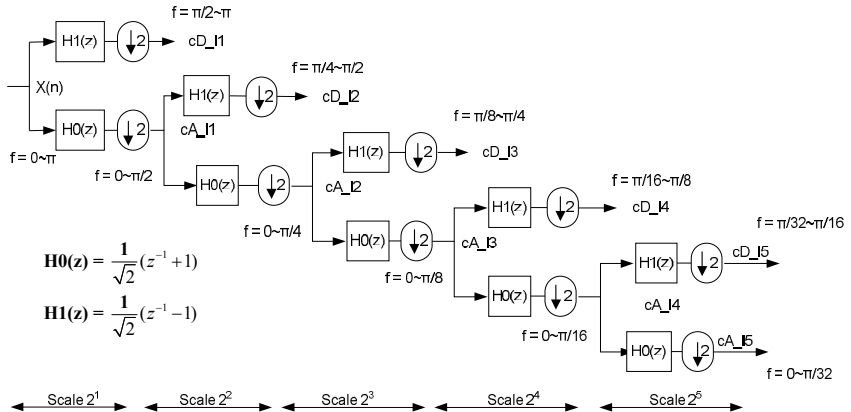

Fig. 4. Cascade filter-bank Implementation of DWT

MMA principle, the boundaries of the constituent ECG waves (P,QRS,T) are expected, due to their morphology (deflection points), to be localized by a modulus-maxima pair (MMP) on the DWT coefficients of the respective scale.

Given the positive or negative deflection of the ECG wave, compared to the isoelectric line, the pair of extrema that indicates the wave's temporal position, can be either a minimum followed by a maximum, for a positive deflection, or the reverse for a negative deflection. Thus the MMA method also allows the characterization of every wave as inverted or not, which is exploited in our algorithm, as illustrated in Fig. 5.

Through MMA, we initially obtain the temporal position of the deflection which demonstrates higher separation from the isoelectric line. This is accomplished by calculating the temporal positions $\left(\mathrm{t}_{1}, \mathrm{t}_{2}\right)$ of the global extrema pair in the cD_13 coefficients. This deflection may correspond to either the R_peak (for a positive deflection) or to Q or S_peak (in case of a negative deflection). Following, MMA is applied in the vicinity of the global extrema pair, in order to obtain a first approximation of the temporal position of the QRS boundaries. The initial estimation of the QRS onset $\left(Q \hat{R} S_{\text {on }}\right)$ is obtained as the preceding extrema ( $\min$ or $\max$ ) from the local MMP in a search window defined as $\left[\mathrm{t}_{1}-4, \mathrm{t}_{1}\right]$. Similarly the offset of the QRS, $\left(Q \hat{R} S_{\text {on }}\right)$ is estimated from the succeeding extrema of the MMP found in $\left[\mathrm{t}_{2}, \mathrm{t}_{2}+4\right]$. Fig. 6 illustrates an example where this procedure is followed to identify the QRS 

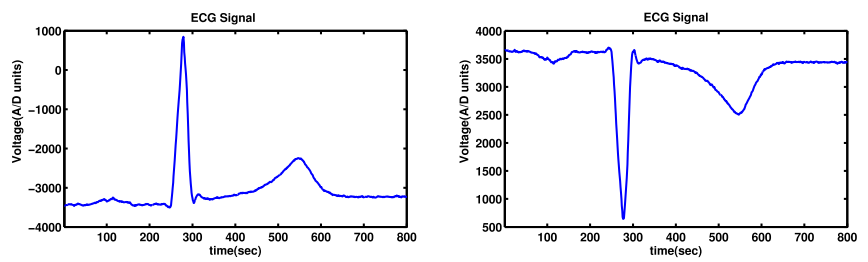

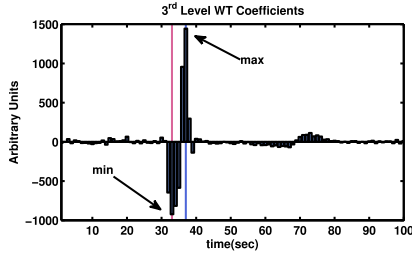

(a) Positive Deflection

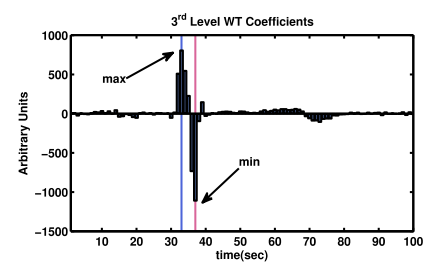

(b) Negative Deflection
Fig. 5. Characterising the direction of the deflection based on the sequence of the WT coefficients extrema pair

boundaries
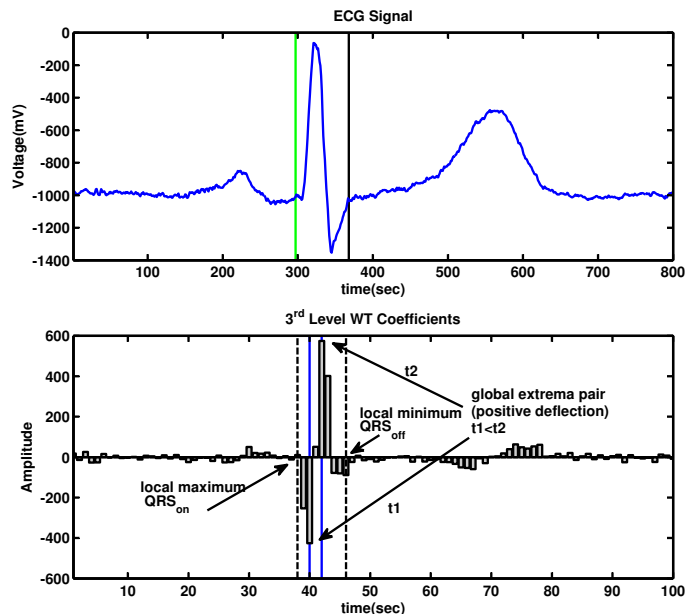

Fig. 6. MMA for the extraction of the QRS boundaries. The global extrema pair localizes the main deflection while the extrema in its vicinity indicate the temporal location of the QRS boundaries

As pointed out earlier, the temporal resolution on the $2^{3}$ scale is diminished (by a factor of 8) compared to the original timescale. This coupled with fact that we operate on a single resolution scale, may inherently lead to less accurate localization of the main deflection (either R_peak or Q, S_peak) and of the QRS boundaries. An example where the MMA produces less accurate results is depicted in Fig. 7. For mitigating that effect we employ a time-domain morphology-based refinement as described in the following subsection.

\section{Time-Domain Morphology (TDM) based Refinement}

The TDM refinement process amends the initial MMA approximation, leading to a more accurate estimation of the QRS fiducial points.

The R_peak time instance is amended first. From the MMA on the $\mathrm{cD} \_13$, we obtain the temporal boundaries $\left(t_{1}, t_{2}\right)$ within which lies the deflection that exhibits higher separation from the isoelectric line. If this deflection is characterized as positive, it is interpreted as an R-wave and thus by projecting $\left(t_{1}, t_{2}\right)$ into the original timescale of $\mathrm{x}[\mathrm{n}]$, the amended

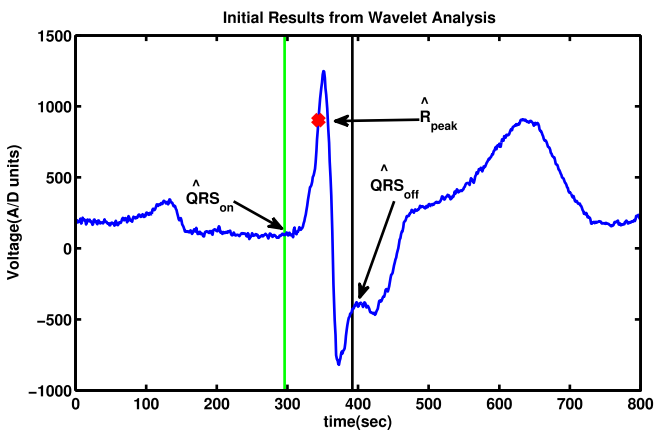

Fig. 7. An example of less accurate estimation of the R_peak and $\mathrm{QRS}_{\text {off }}$ points due to the decreased temporal resolution of scale $2^{3}$ DWT. These results are ameliorated in TDM stage

R_peak time point is calculated as the maximum of the PQRST-complex $(\max (\mathrm{x}[\mathrm{n}]))$ within this time window $(n \in$ $\left.\left[t_{1} \times 2^{3}, t_{2} \times 2^{3}\right]\right)$. If the deflection detected in the MMA is characterized as negative ( $t_{1}$ is a maximum, $t_{2}$ is a minimum) then it corresponds to either the $\mathrm{Q}$ or the $\mathrm{S}$ _peak. Since, the R_peak is always a positive deflection it will be always localized by an MMP with the first point being a minimum and the second a maximum in the $\mathrm{CD}_{-} 13$.

To obtain this pair for this case we have to consider two possible pairs. We thus search before $t_{1}$ for a minimum and after $t_{2}$ for a maximum. The search windows are defined as $\left[t_{1}-15, t_{1}\right]$ and $\left[t_{2}, t_{2}+10\right]$ on the cD_13 timescale. From this we obtain two new ( $t_{a}$-min, $t_{b}$-max) local extrema points in cD_13. From there the two possible pairs of min-max are $\left(t_{a}, t_{1}\right)$ and $\left(t_{2}, t_{b}\right)$. Finally we project the two intervals, $\left[t_{a}, t_{1}\right]$ and $\left[t_{2}, t_{b}\right]$, in the $x[n]$ timescale and obtain the maximum value in each one of them. The final $R$ _peak is chosen as the maximum of the two values.

Obtaining the refinement of the R_peak time instance allows us to focus on the refinement of the QRS boundaries. For this, we exploit the characteristic steep slope of the QRS complex by using the derivative signal. The analysis takes place on the approximate $2^{3}$ scale (cA_13) DWT coefficients. This is chosen from a noise canceling perspective, since we demonstrated that in the $2^{3}$ scale high frequency noise is removed. The initial $\mathrm{QRS}$ boundaries are expanded in time by $64 \mathrm{~ms}$ (equivalent of $8 \mathrm{cD} \_13$ coefficients) before the initial QRS onset $\left(t_{3}=t_{3}-8\right)$ and $120 \mathrm{~ms}$ (equivalent of $15 \mathrm{cD} \_13$ coefficients) after the initial QRS offset $\left(t_{6}=t_{6}+15\right)$. The portion of the ECG signal within these two boundaries is isolated both in $x[n]$ and in $c_{-}$_13[n]. This is performed to ensure that the full QRS-complex is contained in the part of the signal we extract in order to apply the refinement. An approximation of the derivative $f[n]$ is obtained as the backward difference between two successive samples on the $2^{3}$ scale approximate coefficients cA_13. We only calculate $f[n]$ for $n \in\left[\dot{t}_{3}, \dot{t}_{6}\right]$.

To identify the QRS boundaries, the derivative signal is investigated against a predefined threshold and the first time point where the value of the gradient signal becomes higher than the predefined threshold corresponds to the amended QRS boundary. For the $\mathrm{QRS}_{\text {on }}$ the derivative signal is investigated from the beginning up to the amended R_peak time point 
( $\left[t_{3} \rightarrow \mathrm{R} \_\right.$peak $]$) while for the $\mathrm{QRS}_{\text {off }}$, the gradient signal is investigated from its end towards the $R \_p e a k$ ( $\left[R \_p e a k \leftarrow t_{6}\right]$ ). The two thresholds for this operation are defined adaptively and their value is set as a dyadic fraction of the amplitude range of the sampled ECG signal. An example where the TDM amends the MMA results is depicted in Fig. 8. In this case, the initial approximation, (Fig. 7) produced by the DWT analysis is improved after the execution of the TDM stage.

The final step of the QRS refinement includes the approximation of the Q_peak and S_peak time instances. The value for these parameters is extracted as the time instance where the ECG signal, in the original timescale, demonstrates minimum value between the QRS-onset and the R_peak and the R_peak and the QRS-offset for the Q_peak and S_peak respectively. The final outcomes of the amendment process are used in the ultimate stage of the HFEA algorithm which involves the extraction of the clinically relevant features from the $\mathrm{P}$ and T-waves.

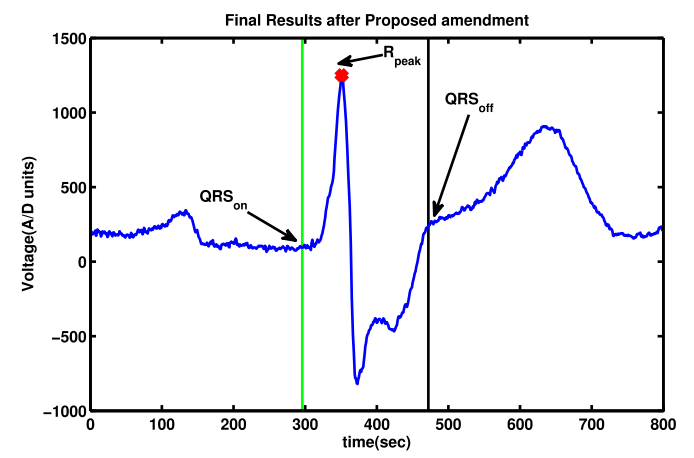

Fig. 8. QRS final estimation on the signal of Fig. 7 after the TDM refinement

\section{P,T-wave feature extraction}

Once the QRS boundaries are finalized after the TDM refinement process, the same modulus-maxima analysis which was applied at the cD_13 is applied at the $2^{5}$ resolution scale detailed coefficients (cD_15) only at the portion of the signal that precedes and succeeds the detected QRS complex in order to identify the $\mathrm{P}$ and $\mathrm{T}$ wave boundaries and the corresponding peaks. These waves are known to demonstrate either convexity or concavity against the isoelectric line and the modulus-maxima pair that localizes the wave, also allows us to characterized it as convex or concave in a similar way that we characterize a deflection as positive or negative. From that point on, the peak of the wave is either the maximum/concave or the minimum/convex point of the original signal $x[n]$ within the modulus-maxima defined wave boundaries. Fig. 9 illustrates the extraction of the fiducial points from the P and Twaves, in the PQRST-complex of Fig. 7. The complete HFEA algorithm is given in the form of pseudocode in Fig. 10. The implementation of the algorithm for validation took place in MATLAB.

\section{COMPUTATIONAL COMPLEXITY AND IMPLEMENTABILITY}

To quantify the complexity of the HFEA algorithm we present here an analytical calculation of the number of arith-

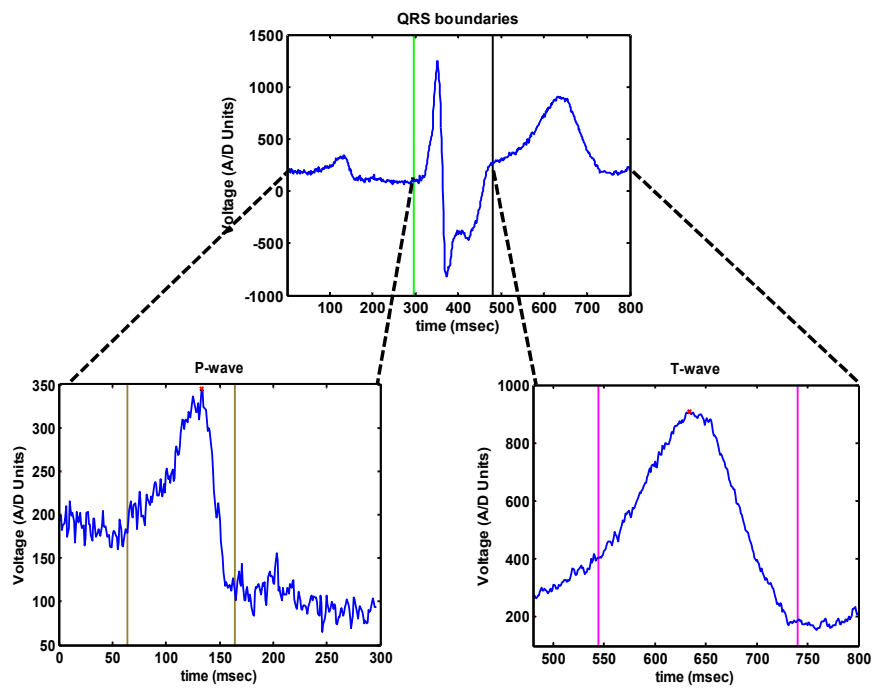

Fig. 9. $\quad \mathrm{P}$ and T-wave feature extraction

metic operations required for the HFEA to run to completion. In principle there are three major components in the HFEA: the DWT coefficients generation, the MMA and the TDM refinement. Following the flow of the pseudocode in Fig. 10, the upper bound of the number of arithmetic operations is derived considering an input of $N$ samples. In our analysis subtractions are considered equivalent to additions, while one comparison operation is considered to have half the complexity of an addition. Comparisons are used in the extraction of extremas $(\min / \max )$ within a specific interval. For the sake of complexity analysis, we consider a completely unfolded architecture with no resource sharing and no optimization, like constant number multiplicatoin, for the operations.

To derive the number of arithmetic operations required for the DWT coefficients generation, we consider that for a single cD_lx or cA_lx coefficient, 1 addition and 1 multiplication is required, according to the Haar DWT high- and low-pass filter transfer functions. In addition, the subsamplers cause each filter output to have half the length of the input. As an example, in order to calculate the cD_11 or cA_11 coefficients individually, $N / 2$ additions and $N / 2$ multiplications are required. Subsequently the calculation of cD_12 or cA_12 requires $N / 4$ additions and $N / 4$ multiplications. In the HFEA we only utilize the cD_13, cA_13 and cD_15 coefficients, which according to Mallat's algorithm, means that from the other resolution scales only cA_11, cA_12 and cA_14 must be calculated. In addition, the cA_15 coefficients do not need to be calculated since cD_15 are computed directly from cA_14. In total, the computational complexity of calculating the coefficients that are employed in the HFEA is $N / 2+N / 4+2 N / 8+N / 16+N / 32$ additions and $N / 2+N / 4+2 N / 8+N / 16+N / 32$ multiplications, or $1.093 \mathrm{~N}$ additions and 1.093 $\mathrm{N}$ multiplications. The MMA step involves the extraction of the max and min value of cD_13, which is of $N / 8$ length. This process requires $(N / 8-1)$ comparisons. The additional MMA calculations in the vicinity of the global MMA pair require 2 additions for the expansion of $t_{1}, t_{2}, 4$ comparisons in the $\left[t_{1}-4, t_{1}\right]$ interval and another 4 comparisons in the $\left[t_{2}, t_{2}+4\right]$ interval (line 9 of Fig. 10), 
1: Initialise

2: Consider a PQRST complex $\mathrm{x}[\mathrm{n}]$ of length $l$

3: HAAR DWT

4: - Calculate DWT Approximate cA_13 and Detailed Coefficients cD_13 and Detailed Coefficients cD_15

\section{5: QRS Initial Estimation}

6: Scale $2^{3}$ MMA

7: Find $t_{1}=\min \left(\mathrm{cD} \_13[\mathrm{n}]\right)$

8: Find $t_{2}=\max \left(\mathrm{cD}_{-} 13[\mathrm{n}]\right)$

9: Find positions $\left(t_{3}, t_{4}\right)$ of the MMP in $\mathrm{cD} \_13[\mathrm{n}]$ for $n \in\left[t_{1}-\right.$ $\left.4, t_{1}\right]$ and $\left(t_{5}, t_{6}\right)$ for $n \in\left[t_{2}, t_{2}+4\right]$

10: Find initial $Q \hat{R} S_{\text {on }}=t_{3} \times 2^{3}$

11: Find initial $Q \hat{R} S_{\text {off }}=t_{6} \times 2^{3}$

\section{2: Time-Domain Refinement (TDM)}

13: if $t_{1}<t_{2}$ then

14: $\quad$ R_peak $=\max (x[n])$, for $n \in\left(t_{1} \times 2^{3}, t_{2} \times 2^{3}\right)$ 15: else

16: Find $t_{a}=\min \left(\mathrm{cD} \_13\right)$, for $\mathrm{cD} \_13 \in\left[t_{1}-15, t_{1}\right]$

17: Find $t_{b}=\max \left(\mathrm{cD}_{-} 13\right)$, for $\mathrm{cD}_{-} 13 \in\left[t_{2}, t_{2}+10\right]$

18: $\quad$ R_peak1 $=\max (\mathrm{x}[\mathrm{n}]), n \in\left[t_{a} \times 2^{3}, t_{1} \times 2^{3}\right]$

19: $\quad$ R_peak2 $=\max (\mathrm{x}[\mathrm{n}]), n \in\left[t_{2} \times 2^{3}, t_{b} \times 2^{3}\right]$

21: end if

22: Set $t h r 1$

23: Set $t h r 2$

24: Expand $t_{3}=t_{3}-8$

25: Expand $t_{6}=t_{6}+15$

26: Approximate Derivative as: $f[n]=c A_{-} l 3[n]-c A_{-} l 3[n-1]$, $n \in\left[\begin{array}{ll}t_{3} & t_{6}\end{array}\right]$

27: Final $Q R S_{\text {on }}$ first $n \in\left[\dot{t}_{3}\right.$ R_peak $], f[n]>t h r 1$

28: Final $Q R S_{\text {off }}$ last $n \in\left[\mathrm{R} \_\right.$peak $\left.t_{6}\right], f[n]>t h r 2$

29: Find Q_peak $=\min (\mathrm{x}[\mathrm{n}]), n \in\left[Q R S_{\text {on }} \times 2^{3} \mathrm{R} \_\right.$peak $]$

30: Find $\mathrm{S} \_$peak $=\min (\mathrm{x}[\mathrm{n}]), n \in\left[\mathrm{R} \_\right.$peak $\left.Q R S_{\text {off }} \times 2^{3}\right]$

31: P,T Wave Estimation

32: Scale $\mathbf{2}^{\mathbf{5}}$ MMA

33: Find $t_{7}=\min \left(\mathrm{cD} \_15[\mathrm{n}]\right), n \in\left[1, Q R S_{o n}\right]$ for $\mathrm{P}$

34: Find $t_{8}=\max \left(\mathrm{cD} \_15[\mathrm{n}]\right), n \in\left[1, Q R S_{\text {on }}\right]$ for $\mathrm{P}$

35: if $t_{7}<t_{8}$ then

36: Calculate $P_{\text {on }}=t_{7} \times 2^{5}$

37: Calculate $P_{\text {off }}=t_{8} \times 2^{5}$

38: $\quad$ P_peak $=\max (\mathrm{x}[\mathrm{n}]) n \in\left[\mathrm{P}_{\text {on }} \mathrm{P}_{\text {off }}\right]$

: else

Calculate $P_{\text {on }}=t_{8} \times 2^{5}$

Calculate $P_{\text {off }}=t_{7} \times 2^{5}$

P_peak $=\min (\mathrm{x}[\mathrm{n}]) n \in\left[\mathrm{P}_{\text {on }} \mathrm{P}_{\text {off }}\right]$

43: end if

44: REPEAT P Block with $n \in\left[Q R S_{\text {off }}\right.$ cD_15_end] for the Twave

Fig. 10. Pseudocode of the HFEA Algorithm

thus in total 6 additions. At this point the TDM refinement step takes place. The $t_{1}, t_{2}$ values, obtained from MMA (line 7,8 of Fig. 10) define an interval of length $T$ in the cD_13 subspace. For the R_peak extraction, the first possibility where $t 1<t 2$, (positive major deflection, line 13 of Fig 10) involves the projection of the $T$ interval boundaries to the original timescale with 2 multiplications, where an interval of length $8 * T$ is now defined and for the extraction of the R_peak $(8 T-1)$ comparisons are required to localize the maximum point and designate it as the $R$ _peak (line 14 of Fig. 10). The second scenario where $t 2<t 1$ (negative major deflection, line 15 of Fig 10 requires 2 additions for the expansion of $t_{1}, t_{2}$ values by 15 and 10 respectively,
$((15-1)+(10-1)=23)$ comparisons for deriving the $t_{a}$ and $t_{b}$ values (lines 16,17 of Fig. 10), 4 multiplications for projecting the $t_{1}, t_{2}, t_{a}, t_{b}$ into the original timescale and a total of $(8 * 15-1)+(8 * 10-1)+1=199$ comparisons for the localization and comparison of the R_peak1 and R_peak2 values (lines 18,19,20 of Fig. 10). The final $R \_p e a k$ is mapped back to $2^{3}$ scale subspace, with 1 multiplication, where it is used for the QRS boundaries refinement beginning with setting the adaptive thresholds, for which $(N+2)$ comparisons, 1 addition and 1 multiplication are required. Assuming that the interval of $\left[t_{3}, t_{6}\right]$ has a length of $M$, after the expansion by 23 samples in total (2 additions, lines 24,25 of Fig. 10), the refinement will take place on a sample of $M+23$ cA_13 coefficients. For the backward difference operation (gradient calculation) in that sample, $(M+22)$ additions are required. The operation of comparing the gradient to the thresholds requires, $M+20$ comparisons for identifying both $\mathrm{QRS}_{\text {on }}$ and $\mathrm{QRS}_{\text {off. }}$ After projecting the QRS boundaries to the original timescale with 2 multiplications, the final stage of the TDM which pertains to the extraction of the $\mathrm{Q}$ and $\mathrm{S}$ _peak position which requires, $8(M+23)-\left(\epsilon_{1}+\epsilon_{2}\right)$ comparisons, where $\epsilon_{1}, \epsilon_{2}$ are the differences in samples in the original timescale between the beginning of the $8(M+23)$ interval and the detected $\mathrm{QRS}_{\text {on }}$ and $\mathrm{QRS}_{\text {off }}$ and the end of the $8(M+23)$ interval respectively (lines 29,30 of Fig 10).

From the above, the TDM stage collectively requires $11 M+$ $N+203-1 / 2\left(\epsilon_{1}+\epsilon_{2}\right)$ comparisons, $M+24$ additions and 6 multiplications, for the first if-statement of the TDM and by following the same process $9 M+N+426-1 / 2\left(\epsilon_{1}+\epsilon_{2}\right)$ comparisons, $M+26$ additions and 8 multiplications for the else-statement. To sum up the calculation of the TDM stage, we consider that $M<N / 8, T<M / 4$ thus $T<N / 32$. Moreover from our experiments $\epsilon_{1}, \epsilon_{2}<30$. With these in mind and by expressing comparisons as half additions the total computational complexity of the TDM stage is $1.31 \mathrm{~N}+95$ additions, 6 mults or $1.18 N+208$ additions, 8 multiplications depending on which if-statement is satisfied.

The final stage of the HFEA for the extraction of the P,Twave fiducial points initially requires the MMA analysis on the cD_15 coefficients. For the P-wave, the MMA can theoretically run on a maximum of $N / 64$ coefficients and may require upto $(N / 64-1)$ comparisons to extract the max and min points (lines 33,34 Fig. 10). Localizing, through MMA, the min and max point of cD_15 defines an interval of length $P$ which after being projected (with 2 multiplications, lines 36,37 Fig. 10) in the original timescale has a length of $8 P$. In this interval the P_peak time instance is localized, as the min or max value of the interval and for this $(8 P-1)$ comparisons are used (line 38 Fig. 10). From our experiments $P<N / 80$, thus the equivalent number of additions for the $\mathrm{P}$-wave analysis is $0.0391 N-1$ additions and 2 multiplications. For the T-wave analysis, the same number of operations is considered to be required, thus the total number for this stage is $0.0875 N-2$ additions and 4 multiplications. From the above investigation the total number of operations required for the HFEA algorithm is $2.553 N+102$ additions and $1.093 N+10$ multiplications, or $2.423 N+214$ additions and $1.093 N+12$ multiplications based on which if-statement is executed on the R_peak extraction. This final 
number represents an upper bound on the required arithmetic operations. It is obvious that the upper bound depends on the number of input samples $N$. By considering the number of multiplications to be approximately the same for both cases, we focus on the number of additions and conclude that for $N \leq 861$ the upper bound is $2.423 N+214$, while for $N>861$ the upper bound is $2.553 N+102$. In reality the number of actual arithmetic operations is going to be lower since $M<<$ $N / 8, T<<N / 32$ and the MMA on cD_15 will be executed on a smaller than $N / 64$ number of coefficients.

We now perform a similar analysis on the computational complexity of the WT-delineator in [34]. In this work, which creates the basis of WT-based ECG delineation, the quadraticspline wavelet is used, with the algorithme 'a trous implementation to avoid decimations. From the transfer functions provided, we observe that for computing a single pair of WT coefficients 4 additions and 4 multiplications are required. Since, the number of generated WT coefficients in each level is the same as the number of input samples, for an input of $N$ samples $4 N$ additions and $4 N$ multiplications are required to generate the WT coefficients in one level. Since the first 5 scales of WT coefficients are considered $17 N$ additions and $17 N$ multiplications are needed for the DWT coefficients generation, if the approximate coefficients of the $2^{5}$ are not computed. It is obvious that the amount of required arithmetic operations only for the calculation of the WT coefficients in [34], without any further processing, is considerably higher that the upper bound of the computational complexity of the HFEA. This is indicative of the possibility for significant power reduction, compared to the WT-delineator of [34], of the HFEA when implemented.

In an attempt to estimate how much energy and silicon area the proposed algorithm may consume, we have coded the HFEA in Verilog and synthesized it using the STMicroelectronics $130 \mathrm{~nm}$ technology library, following the pseudocode of Fig.10 without any further architectural optimization. $1 \mathrm{KHz}$ clock frequency and $1.08 \mathrm{~V}$ supply voltage were used in our synthesis. The synthesized design has an overall cell area of $245 \mathrm{~mm}^{2}$, which is equivalent to $404.7 \mathrm{~K}$ NAND, and consumes $269.64 \mathrm{nW}$ as calculated from Synopsis PrimeTime tool. The number of clock cycles required for the feature extraction operation on a single PQRST complex constituting of 800 samples is approximately 990 clock cycles.

\section{VALIDATION}

\section{A. ECG Databases}

In order to assess the performance of the proposed algorithm and quantify its accuracy, manually annotated ECG signals from various databases are used. Specifically 27 ECG excerpts from the QT database (QTDB), 450 from the PTB database (PTBDB), both available at Physionet [6] as well as 144 samples from the Southampton General Hospital Cardiology (SGHCD) department database were used in our validation.

The QTDB contains 105 two-lead Holter ECG recordings sampled at $250 \mathrm{~Hz}$ of 15 minutes duration, covering a wide range of heart conditions. For each sample the boundaries of the ECG waves (P, QRS, T) and the peaks $(\mathrm{P}, \mathrm{R}, \mathrm{T})$ temporal position are manually annotated by at least one expert and for at least 30 PQRST complexes in each record, resulting in a total of 3622 annotated complexes [36]. The PTBDB contains 549 high resolution $(1 \mathrm{KHz})$ standard 15-lead ECG recordings, with all leads recorded simultaneously from 294 subjects of several different heart conditions (myocardial infarction, hypertrophy, valvular heart diseases, etc.) as well as sinus rhythm. From SGHCD 12 standard 12-lead paper ECG, sampled at frequencies of $250 \mathrm{~Hz}$ and $500 \mathrm{~Hz}$ from patients confirmed to be exhibiting myocardial scar, were digitized at a rate of 1000 samples/s with the use of the ECGScan digitization software [37] and used in our validation.

From the QTDB we chose 27 records and applied the HFEA algorithm on every beat on both ECG channels. We specifically selected those records having annotations for every ECG wave $(\mathrm{P}, \mathrm{QRS}, \mathrm{T})$. The available manual annotations allowed us to partition the signals into individual ECG heartbeats (by considering the $\mathrm{P}_{\text {on }}$ and $\mathrm{T}_{\text {off }}$ ) and then execute the feature extraction algorithm in every one of them. We then calculated the accuracy, in terms of the mean and standard deviation of the error between the algorithm's results and the annotations for each channel separately. Following the guidelines applied in [34], from the two available channels we finally considered the channel that exhibited less error. The overall mean and standard deviation values are calculated as the average mean and standard deviation of the error from the 27 records.

Different to the QTDB, signals from PTBDB and SGHCD were not pre-annotated. From the PTBDB, 30 records were chosen from every available disease category. The 30 ECG records of PTBDB and the 12 records of SGHCD resulted in a total of 450 and 144 heartbeats to be given for annotation respectively. The manual annotations were performed by experts cardiologists with the help of a simple graphical annotator interface also implemented in MATLAB. Whenever the annotation of a particular wave was not possible, either due to the absence of the wave or due to poor sampling, the algorithm's results were disregarded. In order to extract one global temporal position for each fiducial point from the 15 available annotations per record in PTBDB, we followed the $\mathrm{k}$-nearest neighbors (knn) rule applied in [34] for multilead delineators. The 15 onset/offset annotations were ordered in succession and the first time instance which had " $k$ " neighbors within an $e \mathrm{~ms}$ interval was chosen as the wave onset. Conversely, the last time instance obeying in the same knn rule is characterized as the wave offset. We also employed the same multilead rule in the results obtained from our algorithm. For these signals, since only one heartbeat per lead is annotated the mean and the standard deviation were calculated from the error between the annotations and the algorithm's results for the 30 records.

In the SGHCD ECG samples, the 12 leads were captured simultaneously in groups of 3 leads. As a result the previous $k n n$ rule could not be applied. For these signals we followed the CSE guidelines as described in [12]. In every record, the 12 leads were divided into 4 groups each containing the 3 simultaneously captured leads. The groups were formed of leads I-III, aVR-aVF, $\mathrm{V}_{1}-\mathrm{V}_{3}, \mathrm{~V}_{4}-\mathrm{V}_{6}$. For each leadgroup, the earliest onset and the latest offset were selected as the global 
temporal positions of the boundaries of ECG waves. Based on this rule we then calculated the mean and standard deviation between the HFEA results and the manual annotations for each leadgroup separately using all 12 records. The final results were produced by averaging the mean and standard deviation of the 4 leadgroups.

\section{B. Results and Discussion}

Table I lists accuracy results in terms of mean $(\mu)$ and standard deviation $(\sigma)$ attained from executing the HFEA on 27 records from the QTDB. Apart from the mean $(\mu)$ and the standard deviation $(\sigma)$ in $\mathrm{ms}$ we provide the standard deviation in the equivalent number of samples rounded towards the nearest integer. We also list the performance of the quadraticspline based WT delineator, presented in [34] as well as results of the low-pass-differentiator method (LPD) reported in [38], from the same database. The final row lists the tolerance limits for automatic feature extraction algorithms as were defined by the CSE Working party in [5]. Although, in the QTDB results the HFEA algorithm satisfies the CSE tolerance limit only for the T-wave offset, the interpretation of the CSE limits in the QTDB, as also expressed in [34], can not be straightforward due to the limited number of different annotators in the QTDB. Compared to the quadratic-spline WT delineator and the LPD the HFEA demonstrates comparable results, since, only the QRS offset and T-wave peak position are estimated with considerable less accuracy. In the final row we provide the difference of the standard deviations between the HFEA and the CSE tolerance limits in terms of the number of samples, based on the sampling frequency of the database (i.e 1 sample $=4 \mathrm{~ms}$ ). Wherever there is no CSE tolerance we used the lowest standard deviation among the WT delineator by Martinez et. al and LPD. This result was not produced for the P_peak where the HFEA demonstrated the best performance of the three algorithms and there are no CSE tolerance limits. It is to be noted that, for these parameters that the CSE limit is not satisfied, the error, in terms of the actual ECG samples, is one sample, except from T_peak.

Table II lists performance results obtained from the 450 signals of PTBDB and the 144 signals of SGHCD. Both databases facilitated the assessment of the HFEA performance in a multilead ECG system. The knn and the earliest(onset)latest (offset) rules, described in Section V-A, were used to obtain the global temporal position of each parameter for each record from the 15 simultaneously recorded leads of PTBDB and for each leadgroup in SGHCD. In this investigation we restricted ourselves to the parameters, for which CSE tolerance limits are provided and the mutlilead knn rule has been applied previously [34], [39].

Although a different database, PTBDB allows for a more valid comparison with the CSE tolerance limits since it also contains standard 15-lead recordings. The best performance was observed for $k=3$ neighbors for all parameters, and $e=$ $10 \mathrm{~ms}$ for the $\mathrm{P}$-wave boundaries and $\mathrm{QRS}_{\text {on }}$ while $e=12 \mathrm{~ms}$ was used for the $\mathrm{QRS}_{\text {off }}$ and the $\mathrm{T}_{\text {off. }}$. From the results we observe that all CSE tolerance limits are satisfied apart from the one for $\mathrm{QRS}_{\mathrm{on}}$.
The obtained results from the digitized SGHCD database are also given in Table II. The paper digitization process diminished the quality of the actual recordings, thus only one CSE limit $\left(\mathrm{T}_{\text {off }}\right)$ was satisfied in these signals. In spite of this, the outcome of the HFEA algorithm was endorsed by cardiologists.

\section{CONCLUSIONS}

In this paper a novel algorithm (HFEA), based on the combination of WT analysis and time-domain morphology principles, for extracting the ECG fiducial points is proposed. The HFEA is tailored for application in energy constrained remote CVD monitoring environments. The use of DWT with the Haar function as the basis, allows for a significant reduction in the computational complexity compared to other WTbased approaches, which is beneficial in terms of the overall energy consumption. Experiments carried out on ECG signals from publicly available databases, covering both standard 12lead (PTBDB) and ambulatory (QTDB) recordings, as well as on a non-commercial database (SGHCD) show that the HFEA performance is very close to the state of the art ECG delineators. When comparing the HFEA results from PTBDB to the CSE tolerance limits, all but one $\left(\mathrm{QRS}_{\text {on }}\right)$ are satisfied. Although slightly less accurate, the computational complexity, in terms of the required mathematical operations of our approach, which is found to be $2.423 N+214$ additions and $1.093 N+12$ multiplications for $N \leq 861$ or $2.553 N+102$ additions and $1.093 N+10$ multiplications for $N>861$, is significantly smaller than that of the other WT-based approaches. This facilitates the implementation of the HFEA in ultra low-power systems, in the form of a standalone ASIC, enabling the long-term deployment of the HFEA architecture in battery power-devices. By taking into account that mobile CVD monitoring systems are predominantly used for assessing the patient's overall condition, rather than making a complete diagnosis, the proposed scheme is an ideal candidate for the ECG analysis in such systems.

\section{REFERENCES}

[1] Frost \& Sullivan, "Preparing for an aging society: Challengers faced by healthcare system in European Union, Japan and United States," 2009. [Online]. Available: http://www.frost.com/

[2] R. Balani, "Energy Consumption Analysis for Bluetooth, WiFi and Cellular Networks," University of California at Los Angeles, Tech. Rep. TR-UCLA-NESL-200712-01 , Dec, 2007.

[3] Chiron Project, 2010. [Online]. Available: www.chiron-project.eu

[4] F. Rincón and, J. Recas, N. Khaled, and D. Atienza, "Development and Evaluation of Multilead Wavelet-Based ECG Delineation Algorithms for Embedded Wireless Sensor Nodes," IEEE Trans. Inf. Technol. Biomed., vol. 15 , no. 6 , pp. $854-863$, Nov 2011.

[5] The CSE working party, "Recommendations for measurement standards in quantitative electrocardiography," Eur Heart J., vol. 6, no. 10, pp. 815-825, Oct 1985.

[6] A.L. Goldberger et. al., "Physiobank, physiotoolkit, and physionet components of a new research resource for complex physiologic signals," Circulation, vol. 101, no. 23, 2000 (June 13).

[7] M. Nygårds and L. Sörnmo, "Delineation of the QRS complex using the envelope of the E.C.G." Medical and Biological Engineering and Computing, vol. 21, pp. 538-547, 1983.

[8] O. Pahlm and L. Sörnmo, "Software QRS detection in ambulatory monitoring a review," Medical and Biological Engineering and Computing, vol. 22, pp. 289-297, 1984. 


\begin{tabular}{|c|c|c|c|c|c|c|c|c|c|c|}
\hline Algorithm & Feature & $P_{\text {on }}$ & $P_{\text {peak }}$ & $P_{\text {off }}$ & $Q R S_{\text {on }}$ & $R_{\text {peak }}$ & $Q R S_{\text {off }}$ & $T_{\text {on }}$ & $T_{\text {peak }}$ & $T_{\text {off }}$ \\
\hline \hline \multirow{3}{*}{ HFEA } & $\#$ ann. beats & 1620 & 1620 & 1620 & 1620 & 1620 & 1620 & 1620 & 1620 & 1620 \\
\cline { 2 - 12 } & $\mu \pm \sigma(\mathrm{ms})$ & $-6.3 \pm 12.5$ & $5 \pm 9.5$ & $3.1 \pm 16$ & $3.7 \pm 7.8$ & $3.8 \pm 9.8$ & $12.1 \pm 16.6$ & $-15.8 \pm 34$ & $-15.3 \pm 29.3-16.6 \pm 20.8$ \\
\cline { 2 - 12 } & $\sigma$ (samples) & 3 & 2 & 4 & 2 & 2 & 4 & 8 & 7 & 5 \\
\hline \multirow{2}{*}{$\begin{array}{c}\text { Martinez } \\
\text { et.al. }[34]\end{array}$} & $\#$ ann. beats & 3194 & 3194 & 3194 & 3623 & - & 3623 & - & 3542 & 3542 \\
\hline \multirow{2}{*}{ LPD [38] } & $\mu \pm \sigma(\mathrm{ms})$ & $2.0 \pm 14.8$ & $3.6 \pm 13.2$ & $1.9 \pm 12.8$ & $4.6 \pm 7.7$ & N/A & $0.8 \pm 8.7$ & N/A & $0.2 \pm 13.9$ & $-1.6 \pm 18.1$ \\
\cline { 2 - 11 } & $\mu$ ann. beats & 3194 & 3194 & 3194 & 3623 & - & 3623 & - & 3542 & 3542 \\
\hline CSE [5] & $2 \sigma(m s)$ & $14 \pm 13.3$ & $4.8 \pm 10.6$ & $-0.1 \pm 12.3$ & $-3.6 \pm 8.6$ & N/A & $-1.1 \pm 8.3$ & N/A & $-7.2 \pm 14.3$ & $13.5 \pm 27.0$ \\
\hline diff of $\sigma$ & (samples) & 10.2 & - & 12.7 & 6.5 & - & 11.6 & - & - & 30.6 \\
\hline
\end{tabular}

TABLE I

Feature Extraction Performance Results From QTDB

\begin{tabular}{|c|c|c|c|c|c|c|}
\hline Database & Feature & $P_{\text {on }}$ & $P_{\text {off }}$ & $Q R S_{\text {on }}$ & $Q R S_{\text {off }}$ & $T_{\text {off }}$ \\
\hline \hline \multirow{2}{*}{ PTBDB } & $\#$ ann. beats & 422 & 422 & 450 & 450 & 432 \\
\cline { 2 - 7 } & $\mu \pm \sigma(\mathrm{ms})$ & $1.1 \pm 9.5$ & $-6 \pm 11$ & $3.8 \pm 10.8$ & $3.7 \pm 6.8$ & $-8 \pm 10.8$ \\
\hline \multirow{2}{*}{ SGHCD } & $\#$ ann. beats & 128 & 128 & 144 & 144 & 132 \\
\cline { 2 - 7 } & $\mu \pm \sigma(\mathrm{ms})$ & $8.8 \pm 14.4$ & $-1.8 \pm 15.7$ & $4.1 \pm 16.2$ & $6 \pm 14.3$ & $-3.8 \pm 21.3$ \\
\hline CSE [5] & $2 \sigma$ & 10.2 & 12.7 & 6.5 & 11.6 & 30.6 \\
\hline
\end{tabular}

TABLE II

Feature Extraction PERformance Results From PTBDB AND SGHCD

[9] J. Pan and W. J. Tompkins, "A real-time QRS detection algorithm," IEEE Trans Biomed Eng, vol. 32, no. 3, pp. 230-236, Mar 1985.

[10] P. S. Hamilton and W. J. Tompkins, "Quantitative investigation of QRS detection rules using the MIT/BIH arrhythmia database." IEEE Trans. Biomed. Eng., vol. 33, no. 12, pp. 1157-1165, Dec 1986.

[11] L. Sörnmo, "A model-based approach to QRS delineation," Comput. Biomed. Res., vol. 20, pp. 526-542, Dec 1987.

[12] J. Willems et al., "Assessment of the performance of electrocardiographic computer programs with the use of a reference data base," Circulation, vol. 71, no. 3, pp. 523-5, Mar 1985.

[13] F. Gritzali, "Towards a generalized scheme for QRS detection in ECG waveforms," Signal Process., vol. 15, pp. 183-192, September 1988.

[14] Z. Dokur, T. Olmez, M. Korurek, and E. Yazgan, "Detection of ECG waveforms by using artificial neural networks," in Proc. IEEE EMBS, vol. 3, oct-3 nov 1996, pp. 929 -930 vol.3.

[15] E. Soria-Olivas et al., "Application of adaptive signal processing for determining the limits of $\mathrm{P}$ and $\mathrm{T}$ waves in an ECG," IEEE Trans. Biomed. Eng., vol. 45, no. 8, pp. 1077 -1080, Aug 1998.

[16] R. V. Andreão, B. Dorizzi, and J. Boudy, "ECG signal analysis through Hidden Markov Models," IEEE Trans. Biomed Eng, vol. 53, no. 8, pp. 1541-1549, Aug 2006.

[17] Z. Yong, H. Wenxue, X. Yonghong, and C. Jianxin, "ECG Beats Classification Based on Ensemble Feature Composed of Independent Components and QRS Complex Width," in International Conference on Computer Science and Software Engineering, vol. 1, Dec 2008, pp. 868 $-871$.

[18] M.-Y. Yang, W.-C. Hu, and L.-Y. Shyu, "ECG events detection and classification using wavelet and neural networks," in Proc. IEEE EMBS, vol. 1, Oct-2 Nov 1997, pp. $289-292$.

[19] Z. Dokur, T. Olmez, and E. Yazgan, "ECG waveform classification using the neural network and wavelet transform," in Proc. IEEE EMBS, vol. 1, 1999, p. 273 vol.1.

[20] S. Szilágyi and L. Szilágyi, "Wavelet Transform and Neural-Network Based Adaptive Filtering for QRS Detection," in Proc. IEEE EMBS, vol. 1, Jul 2000.

[21] S. Szilagyi, Z. Benyo, L. Szilagyi, and L. David, "Adaptive wavelettransform-based ECG waveforms detection," in Proc. IEEE EMBS, vol. 3, sept. 2003, pp. $2412-2415$ Vol.3.

[22] R. V. a. Andreão and J. Boudy, "Combining wavelet transform and hidden Markov models for ECG segmentation," EURASIP J. Appl. Signal Process., vol. 2007, pp. 95-95, Jan 2007.

[23] G. de Lannoy, B. Frenay, M. Verleysen, and J. Delbeke, "Supervised ECG delineation using the wavelet transform and hidden Markov models," in Proc. IFMBE, vol. 22, 2008, pp. 22-25.

[24] C. Li, C. Zheng, and C. Tai, "Detection of ECG characteristic points using wavelet transforms," IEEE Trans. Biomed. Eng., vol. 42, no. 1, pp. $21-28$, Jan 1995.

[25] S. Kadambe, R. Murray, and G. Boudreaux-Bartels, "The dyadic wavelet transform based QRS detector [ECG analysis]," in Twenty-Sixth Asilomar Conference on Signals, Systems and Computers, Oct 1992, pp. 130 -134 vol.1.

[26] V. Di Virgilio, C. Francaiancia, S. Lino, and S. Cerutti, "ECG fiducial points detection through wavelet transform," in Proc. IEEE EMBS, vol. 2, sep 1995, pp. $1051-1052$ vol.2.

[27] J. Sahambi, S. Tandon, and R. Bhatt, "Using wavelet transforms for ECG characterization. An on-line digital signal processing system," IEEE Eng Med. Biol Mag, vol. 16, no. 1, pp. 77 -83, Jan.-Feb 1997.

[28] M. Bahoura, M. Hassani, and M. Hubin, "DSP implementation of wavelet transform for real time ECG wave forms detection and heart rate analysis," Computer Methods and Programs in Biomedicine, vol. 52, no. 1 , pp. $35-44,1997$.

[29] S. Mahmoodabadi, A. Ahmadian, M. Abolhasani, M. Eslami, and J. Bidgoli, "ECG Feature Extraction Based on Multiresolution Wavelet Transform," in Proc. IEEE EMBS, Jan 2005, pp. $3902-3905$.

[30] K. Ouni, S. Ktata, and N. Ellouze, "Automatic ECG segmentation based on Wavelet Transform Modulus Maxima," in IMACS, vol. 1, Oct 2006, pp. $140-144$.

[31] A. Josko, "Discrete Wavelet Transform In Automatic ECG Signal Analysis," in IEEE Instrumentation Measurement Technology Conf., may 2007, pp. $1-3$.

[32] Y. Velchev and O. Boumbarov, "Wavelet Transform Based ECG Characteristic Points Detector," in Intl. Scientific Conf. Comp. Science, vol. 1, 2008, pp. 22-25.

[33] J. Martinez, S. Olmos, and P. Laguna, "Evaluation of a waveletbased ECG waveform detector on the QT database," in Computers in Cardiology, 2000, pp. $81-84$.

[34] J. Martinez, R. Almeida, S. Olmos, A. Rocha, and P. Laguna, "A wavelet-based ECG delineator: evaluation on standard databases," IEEE Trans. Biomed. Eng., vol. 51, no. 4, pp. 570 -581, Apr 2004.

[35] A. Burns et al., "Shimmer ${ }^{\mathrm{TM}}$ - a wireless sensor platform for noninvasive biomedical research," IEEE Sensors J., vol. 10, no. 9, pp. 1527 -1534, Sep 2010.

[36] P. Laguna, R. Mark, A. Goldberg, and G. Moody, "A database for evaluation of algorithms for measurement of QT and other waveform intervals in the ECG," in Computers in Cardiology, Sep 1997, pp. 673 $-676$.

[37] F. Badilini, T. Erdem, W. Zareba, and A. Moss, "ECGScan: a method for conversion of paper electrocardiographic printouts to digital electrocardiographic files." J Electrocardiol, vol. 38, no. 4, pp. 310-8, Oct 2005.

[38] P. Laguna, R. Jané, and P. Caminal, "Automatic detection of wave boundaries in multilead ECG signals: validation with the CSE database," Comput Biomed Res., vol. 27, pp. 45-60, Feb 1994.

[39] P. de Chazal and B. Celler, "Automatic measurement of the QRS onset and offset in individual ECG leads," in Proc. IEEE EMBS, vol. 4, 1996. 


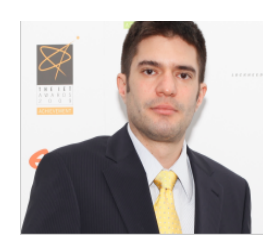

estimation algorithms.
Evangelos B. Mazomenos obtained a Diploma (5year) in Electrical \& Computer Engineering from the Univ. of Patras, Greece and a PhD from the School of ECS, Univ. of Southampton, UK in 2006 and 2012 respectively. He is the recipient of the 2009 IET Leslie H. Paddle fellowship on post-graduate studies for his $\mathrm{PhD}$ research on real-time target tracking in WSN. From January 2011 he has been a research fellow in ECS. His research interests are in the area of WSNs with a focus on positioning and tracking, biomedical signal processing and dynamic

Dwaipayan Biswas obtained his MSc, in System on Chip, 2010-2011 from the University of Southampton, Southampton, UK. He is presently a $\mathrm{PhD}$ student working on Biomedical Engineering with the Electronics and Software Systems group at the University of Southampton. His research areas include Biomedical signal processing and low power VLSI design.

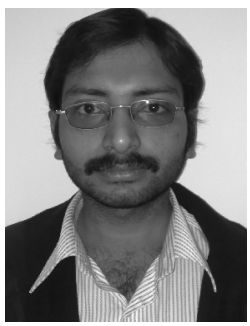

Amit Acharyya received the Ph.D. degree in 2011 from the School of Electronics and Computer Science in the University of Southampton, UK. Currently he is working as an Assistant Professor in the Indian Institute of Technology (IIT), Hyderabad, India. His research interests include signal processing algorithms, VLSI architectures, low power design techniques, computer arithmetic, numerical analysis, linear algebra, bio-informatics and electronic aspects of pervasive computing.

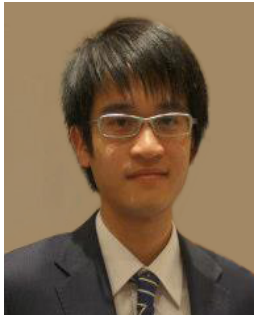

Taihai Chen received the B.Eng. degree (1st Class Hons.) from University of Electronic Science and Technology of China (UESTC), Chengdu, China in 2009, and later received the M.Sc. degree (Distinction) from University of Southampton, Southampton, UK in 2010. Since October 2010, he has been working towards his Ph.D degree in Electronic and Software Systems (ESS) research group in University of Southampton. His research interests include biomedical signal processing, low-power design techniques, pattern recognition, machine learning, associated VLSI architectures, and next-generation healthcare systems.

Koushik Maharatna (M02) received the M.Sc. degree in electronic science from Calcutta University,

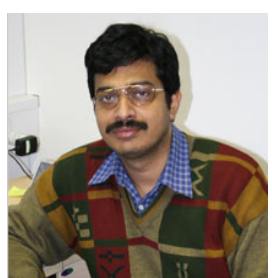
and Computer Science of the University of Southampton, U.K., where he is currently a Reader. His research interests include low-power VLSI and signal processing for applications in DSP, communication and next-generation healthcare systems, computer arithmetic, analog signal processing, and bioinspired circuits and systems. Dr. Maharatna is a member of the IEEE VLSI System Application (VSA) Technical Committee.

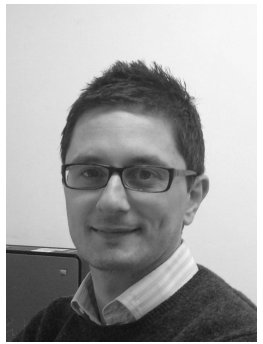

James Rosengarten received his MBBS from the University of London in 2002 and became a member of of the Royal College of Physicians (MRCP(UK)) in 2005. Currently he is a clinical cardiologist based in Southampton. Following training in general cardiology in London and Wessex, he is now specialising in heart rhythm disorders, including invasive testing and implantable devices. He is currently the Research Fellow in Cardiac Rhythm Management, based at University Hospital Southampton. Under the supervision of Prof. Morgan and Prof. Hanson at the University of Southampton, he is utilising engineering techniques to discover novel biomarkers of sudden cardiac death risk.

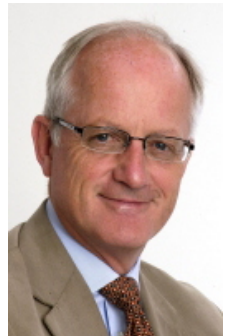

John Morgan graduated from the University of Cambridge with a BA in Natural Sciences in 1979 and from the Westminster Medical School with an MB BChir in 1982. He then obtained an MA and an MD from Cambridge University in 1983 and 1991 respectively. He became a member of the Royal College of Physicians (MRCP(UK)) in 1985 and Fellow (FRCP) in 1995. On his appointment as Consultant Cardiologist, Wessex Cardiothoracic Centre (1992), he founded the Wessex Cardiac Arrhythmia Management Service which has become recognised as a centre of clinical and research excellence. He was appointed an honorary professor at the University of Teesside in 2006 for his work in medical education and received a personal chair in the School of Medicine, University of Southampton in 2007. Professor Morgan holds several published patents relating to invention of novel interventional and device technologies. He supervises/has supervised several fellows in doctoral research projects and continues to research and publish extensively and is an internationally recognised leader in his field. He also sits on a series of national and international boards/professional associations and governing bodies.

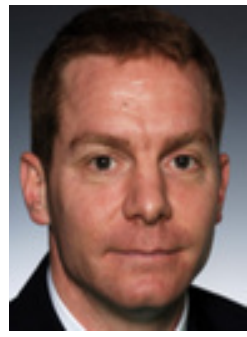

Nick Curzen is a Consultant Cardiologist at University Southampton Hospitals and Professor of Interventional Cardiology at the University of Southampton. Betwenn 1993-1996 he trained in cardiology at Southampton, Bournemouth, Royal Brompton, London Chest and St Bartholomews hospitals and completed a $\mathrm{PhD}$ in vascular biology at Imperial College, London. He has wide ranging research interests including platelet function, myocardial ischaemia, cardiac magnetic resonance and scar. $\mathrm{He}$ has over 130 peer review scientific papers and has edited 3 cardiology textbooks. In November 2010, Nick Curzen came top of a national poll of interventional cardiologist published in the Daily Mail. 